\title{
Ligand-Binding Assay Development: What Do You Want to Measure Versus What You Are Measuring?
}

\author{
Andrew P. Mayer ${ }^{1,2,3}$ (D) and Charles S. Hottenstein ${ }^{1,2}$ \\ Received 9 November 2015; accepted 4 December 2015; published online 15 December 2015
}

KEY WORDS: characterize; free/total; immunoassay; ligand-binding assays.

\section{INTRODUCTION}

The analysis of biotherapeutics by ligand-binding assay (LBA) is associated with some unique challenges that are unlike those commonly encountered in chromatographic methods for chemically based small molecule drugs. While small molecule drugs are typically highly protein bound, the use of an extraction procedure disrupts these interactions and thus allows for measurement of total drug concentrations; modeling is subsequently required to estimate the amount of free drug based upon protein-binding data. Protein-binding interactions for small molecule drugs are typically low affinity and high capacity in nature (i.e., albumin binding). In contrast, LBAs are most often used for the quantitation of large molecule proteins or peptides, where the use of an extraction procedure is replaced by sample dilution. Interferences in ligand-binding assays are typically of high affinity and low capacity and include target, receptors, antidrug antibodies, and binding proteins $(1,2)$, however some are typically low affinity such as anti-drug antibodies. While ligandbinding assays are traditionally the methodology of choice for the quantification of biotherapeutics in biological matrices, there is a trend towards increased use of chromatographic methods. Immunoenrichment and enzymatic digestion followed by the LC-MS/MS monitoring of specific biotherapeutic peptide fragments for use as a surrogate to quantify the whole molecule are becoming emergent techniques in the bioanalyst toolbox $(3,4)$. This diversity in analytical technology often times leads to goodnatured debate among bioanalytical scientists who tend to gravitate towards one of the two technological approaches. Yet, during assay development, there are two critical questions that often go unanswered; what do you want to measure and what are you measuring?

\footnotetext{
${ }^{1}$ GlaxoSmithKline Pharmaceuticals Research and Development, 709 Swedeland Road, King of Prussia, Pennsylvania 19406, USA.

${ }^{2}$ Platform Technology and Science, DMPK-Bioanalytical Science and Toxicokinetics, King of Prussia, Pennsylvania, USA.

${ }^{3}$ To whom correspondence should be addressed. (e-mail: andrew.p.mayer@gsk.com; )
}

\section{WHAT DO YOU WANT TO MEASURE?}

Increased attention has been given to the form of the drug being measured, whether it is "free," "total," or "bound" (5-11). These fractions can be difficult to quantitate to an absolute measure, making it important to properly design and characterize the assay. Care must be taken to understand how the analytical method ultimately perturbs the in vivo equilibrium, which may not be a true representation of that environment. Binding different regions of the biotherapeutics via the capture reagent whether target, anti-idiotypic antibody, or to a more generic capture mechanism (protein A etc.) determines which form of the drug is being captured and ultimately measured. Utilizing the drug target or neutralizing anti-idiotypic antibody against the binding portion of the molecule will presumably only capture the "free" or monovalently bound form of the drug (if the drug is an antibody). Antigen and ADA-challenge experiments, where antigen and the surrogate ADA are spiked into control plasma with drug, can help to characterize the assay limits where quantitation of drug cannot be accurately measured in the presence of antigen or ADA. A non-neutralizing anti-idiotypic reagent will bind to the drug outside of the CDR region (for an antibody), allowing both monovalently and bivalently bound forms of the drug to be measured. There are a number of ways to capture a drug (monoclonal antibody), including Protein A, Protein G, and Protein L, which interact with the $F_{c}$ of humanized $\mathrm{mAbs}$ and can be a useful generic mechanism to capture the drug independent of target binding. In addition to the "free" form of the drug, various heterogeneous forms of the drug can be detected as a result of in vivo exposure. Molecular variants and enzymatic catabolism products may have the potential to be detected dependent upon the specificity of the capture and detection antibodies utilized in the assay. Potential catabolism products, as well as deamidation, oxidation, glycation, and/or aggregation species, might be measureable in a given assay, however, it may not represent an active drug. Similar questions can be asked of proteins and peptides, which will undergo metabolism and "clippage" by proteases in vivo. Are the reagents being used in the assay measuring the "total" peptide (active plus inactive) or 
are they quantitating only the pharmacologically active portion of the molecule? The answer to "what do we want to measure" should always be primary, as the selection of critical reagents and technological platforms dictates what form of the drug is measured and data interpretation is dependent on knowing these details. This question needs input from data users: be it pharmacokinetic scientists, clinicians, or other key stakeholders in order to drive platform and reagent selection. Given a combination of "total" or "free" drug and target concentrations, our modeling colleagues can help to estimate the "free," "bound," and/or "total" concentrations of drug. This leads to a paradigm shift in how the industry has traditionally viewed assay development and validation; although we might have a robust assay with excellent precision and accuracy, if we are not measuring the desired form of the drug data generated could lead to incorrect interpretation whether it be safety, for PK/PD translation, or discharging program risk (5). Furthermore, the analysis of samples by a valid assay does not necessarily lead to data with true scientific understanding. As bioanalytical scientists, we need to be intricately involved in our assay design, knowing the advantages and limitations of the reagents we employ, with the "end result" of each study in mind. The exercise of bioanalytical method development and validation should never be a "one size fits all" approach, as the data the assay generates depend on the questions the data will answer. Studies with a PK/PD efficacy primary endpoint often require an assay measuring available pharmacologically active drug, as this is typically the fraction of the circulating drug which is believed to drive the mechanism of action. Studies with a safety endpoint (GLP Toxicology etc.) might require an assay measuring "total" drug, to determine if the drug is accumulating and potentially circulating longer than the available pharmacologically active portion which may affect known safety margins.

\section{WHAT ARE YOU MEASURING?}

The second important question which needs to be answered is "what are we measuring." The equilibrium that exists within the primary step of any assay in conjunction with the reaction kinetics due to specific assay conditions where the drug and target ligand are in flux between the "free" and "bound" state are tenuous. Changing certain assay conditions can drive this equilibrium towards the "bound" ("product") or "free" ("reactants") state.

$\mathrm{mAb}+L \Leftrightarrow \mathrm{mAb} \times L$

$K_{d}=\frac{1}{K_{a}}=\frac{|\mathrm{mAb}|_{\text {free }} \times|L|_{\text {free }}}{|\mathrm{mAb} \times L|_{\text {bound }}}$

Analyzing samples utilizing an assay designed to measure pharmacologically active and available drug does not necessarily ensure the data represents the "free" fraction. Receptor shedding, target interference, anti-drug antibodies, drug catabolism, deamidation, aggregation, and a host of other in vivo modifications to either drug or target can affect the end measurement. Assay validation should be an iterative process where characterization of an assay increases as a drug moves down the pipeline towards approval. That is to say that the extent of characterization for an assay to support a non-clinical study should be less than one to support a Phase 3 clinical trial or BE study. The underlying biology and drug mechanism of action are important to understand in determining assay interferences which may need to be overcome. Careful consideration must be given when characterizing any assay as "free," "bound," or "total". These terms are often used without proper assay characterization. In practice, it is difficult to measure the true "free" or "bound" in vivo state of any molecule. It should be noted that while the form of the drug (free or bound) is in a static state in the specimen tube, this equilibrium is perturbed and changes as soon as it is processed for analysis. While assay specific reagents play an important role in determining the form of the drug that is measured, assay conditions play an equally important role. The farther this equilibrium is pushed towards the products, the more "total" the assay becomes. The conditions of the assay are integral to what the assay measures. This includes the concentration of the capture reagent, minimum required dilution, the length of primary incubation time, incubation temperature, and assay buffer constituents which can all have an effect on assay equilibrium. The analytical platform used can also impact the form of he drug being measured but can also be important when attempting to determine which form of the drug is being measured. High resolution mass spectrometry (HR-MS) has the ability to determine if the form of the drug being measured has undergone some form of biotransformation (12). Both HR-MS and traditional LC-MS/MS can be used as an orthogonal tool in combination with the primary immunoassay method to confirm or refute the free or total form of the drug claimed to be measured (13). During method development, an understanding needs to be cultivated as to how each of these variables affects the recovery of drug in real samples. During validation, understanding the effect of target interference and anti-drug antibodies (by utilizing a surrogate control) should be evaluated at a minimum. As bioanalytical scientists, we must strive to fully characterize our assays, to understand their strengths and limitations (14). While these experiments require time and often go above and beyond current regulatory requirements, the result will be robust bioanalytical methods with easily translatable and defendable data.

\section{CONCLUSION}

Scientists need to continue to characterize their assays throughout the development process, as to better understand the strengths and limitations of their assays and how the results should be interpreted. We as bioanalytical scientists need to continue to engage the end user of the data, so that we provide quality data that helps to answer the questions at hand but only after we begin by answering the two most primary and fundamental questions: "what do we want to measure" and "what are we measuring"?

\section{REFERENCES}

1. Findlay JWA. Specificity and accuracy data for ligand-binding assays for macromolecules should be interpreted with caution. AAPS J. 2008;3:433-4.

2. Findlay JWA. Specificity of bioanalytical methods for pharmacokinetics support of biological macromolecular therapeutics is important. Bioanalysis. 2011;3(6):567-9.

3. Zheng J, Mehl J, Zhu Y, Xin B, Olah T. Application and challenges in using LC-MS assays for absolute quantitative analysis of therapeutic proteins in drug discovery. Bioanalysis. 2014;6(6):859-79. 
4. Palandra J, Finelli A, Zhu M, Masferrer J, Neubert H. High specific and sensitive measurement of human and monkey interleukin 21 using sequential protein and tryptic peptide immunoaffinity LC-MS/MS. Anal Chem. 2013;85(11):5522-9.

5. Lee JW, Kelley M, King LE, Yang J, Salimi-Moosavi H, Tang M, et al. Bioanalytical approaches to quantify "Total" and "Free" therapeutic antibodies and their targets: technical challenges and PK/PD applications over the course of drug development. Bioanalysis. 2011;13(1):99-110.

6. Kuang B, King L, Wang HF. Therapeutic monoclonal concentration monitoring: free or total? Bioanalysis. 2010;2(6):1125-40.

7. Kelley M, Ahene AB, Gorovits B, Kamerud J, King L, McIntosh $\mathrm{T}$, et al. Theoretical considerations and practical approaches to address the effect of anti-drug antibody (ADA) on quantification of biotherapeutics in circulation. AAPS J. 2013;15(3):646-58.

8. Yang J, Quarmby V. Free versus total ligand-binding assays: points to consider in biotherapeutic drug development. Bioanalysis. 2011;3(11):1163-5.

9. Heinig K, Wirz T, Schick E, Guenzi A. Bioanalysis of therapeutic peptides: differentiating between total and anti-drug antibody bound using liquid chromatography-tandem mass spectrometry quantitation. J Chromatogr A. 2013;1316:69-77.

10. Verch T, Chilewski S, Bouaraphan S, Yarovoi H, Yin KC, Chen $\mathrm{D}$, et al. Pharmacokinetic immunoassay methods in the presence of soluble target. J Immunol Methods. 2010;361:7581.

11. Williams L, Sank M, Chimalakonda A, Ni Y, Saewert M, DeSilva $\mathrm{B}$, et al. Development and characterization of a free therapeutic ligand binding assay with assistance from kinetics modeling. J Immunol Methods. 2015;419:18-24.

12. Hall MP. Biotransformation and in vivo stability of protein biotherapeutics: impact on candidate selection and pharmacokinetic profiling. Drug Metab Dispos. 2014;42:1873-80.

13. Lee JW. Orthogonal tools to help determine the required selectivity of ligand-binding assays in drug development. Bioanalysis. 2014;6(8):1037-40.

14. Stevenson L, Amaravadi L, Myler H, Salazar-Fontana L, Gorovits B, Kirshner S, et al. White paper on recent issues in bioanalysis: a full immersion in bioanalysis (Part 3 - LBA and immunogenicity). Bioanalysis. 2014;6(24):3355-68. 